the training of nurses in sexual and reproductive health (SRH) across the UK and it is preferable that this should be accredited by the Faculty of Sexual and Reproductive Healthcare (FSRH).

There is also the valuable point that there is a belief that accreditation can only be achieved through academic institutes and the Royal College of Nursing $(\mathrm{RCN})$ ! This is usually at significant cost for the course, time out of practice and the cost of replacing that member of staff for the duration of the course; consequently not cost effective in these times of ever-reducing budgets. The application for accreditation from the RCN is now very expensive. The nurses that have had the enthusiasm and dedication to train in fitting implants and intrauterine devices/ systems (which increases the flexibility of the SRH service and improves service user choice) need to have the opportunity to access accreditation.

I would support the notion that all nurses who have undertaken the SRH course and extended training in these areas should be able to obtain accreditation from the FSRH and therefore ensure that their skills are transferable. I would be very happy to offer any assistance and support to move this initiative forward.

Adrienne Swarbrick, BSC, RN

Advanced Nurse Practitioner, CaSH Service, Lancashire Care NHS Foundation Trust, UK; adrienne.swarbrick@lancashirecare.nhs.uk

Competing interests None.

J Fam Plan Reprod Health Care 2013;39:67. doi:10.1136/jprhc-2012-100488

\title{
REFERENCE
}

1 Mehighan S, Burnett J. An innovative training for nurses in sexual and reproductive health. J Fam Plann Reprod Health Care 2012;38:194-195.

\section{Nurse accreditation: a better way of working}

Well done Berkshire. I completely agree with the sentiments expressed by Mehigan and Burnett in their recent article in the Journal. ${ }^{1}$ It is essential that some standardisation is needed in 